\title{
New therapeutic target identified for Alzheimer disease
}

US-based researchers have identified a protein that selectively regulates $\gamma$-secretase generation of amyloid- $\beta$ $(\mathrm{A} \beta)$, a peptide implicated in the pathogenesis of Alzheimer disease (AD). The newly identified regulatory molecule $-\gamma$-secretase-activating protein (GSAP) - might constitute an A $\beta$ lowering therapeutic target, according to the investigators.

$\gamma$-Secretase inhibition represents a promising therapeutic strategy for AD. "However, one problem with inhibiting this enzyme is that it also has other targets, notably Notch, which is essential for normal biological function," explains Paul Greengard, principal investigator of the new study.

Greengard and his colleagues set out to further understand how $\gamma$-secretase achieves substrate specificity. The researchers had previously demonstrated that imatinib, an anti-cancer drug, could inhibit $\gamma$-secretase cleavage of the amyloid precursor protein C-terminal fragment, from which $A \beta$ is derived, while seemingly having no effect on Notch processing. Given these findings, the first aim of the new study was to identify the molecular target of imatinib that conferred the drug's effect on $A \beta$ production.

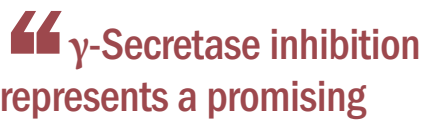
therapeutic strategy for AD 77

In a series of experiments using membrane or whole-cell preparations, derivatives of imatinib were consistently found to associate with a $16 \mathrm{kDa}$ protein that, in turn, bound components of $\gamma$-secretase. Further analyses revealed that the $16 \mathrm{kDa}$ molecule was the predominant form of a widely conserved yet uncharacterized protein, which the investigators went on to name GSAP.

The researchers examined the effect of altering GSAP levels on $A \beta$ production in cell-based and in vitro $\gamma$-secretase assays. In these experiments, lowering the expression of GSAP inhibited generation of $A \beta$, while increasing the expression of this protein led to a rise in $A \beta$ levels. By contrast, similar assays assessing Notch cleavage revealed that GSAP had no effect on this $\gamma$-secretase activity.

To examine whether GSAP could affect AD pathology in vivo, the researchers generated a mouse model of AD in which the expression of GSAP could be lowered. In such mice, long-term knockdown of GSAP led to a marked reduction in amyloid plaque load in the absence of pathological changes indicative of impaired Notch signaling.

The researchers believe that the development of compounds that target GSAP could lead to disease-modifying therapies for AD. They stress, however, that such compounds (unlike imatinib, which is rapidly pumped out of the brain) must be able to accumulate in the brain.

Darran Yates

Original article He, G. et al. Gamma-secretase activating protein is a therapeutic target for Alzheimer's disease. Nature 467, 95-98 (2010) 